\title{
Network model for the numerical solution of groundwater flow: Application to partially penetrating retaining structures in geotechnical engineering
}

\author{
Encarnación Martínez-Moreno | Iván Alhama Manteca | Gonzalo García-Ros@
}

Higher Technical School of Civil Engineering, Technical University of Cartagena Cartagena, Spain

\section{Correspondence}

Gonzalo García-Ros, Higher Technical School of Civil Engineering, Technical University of Cartagena, Paseo Alfonso XIII, 52, 30203 Cartagena, Spain. Email: gonzalo.garcia@upct.es

MSC2000: 35J05, 35C99
Based on the network simulation method, a precise numerical model is designed for the 2-D groundwater flow in porous and isotropic aquifers. If a partially penetrating impervious barrier exists, groundwater will flow downstream circumventing the underground structure. The network model is solved numerically in a circuit simulation free code. Due to the powerful mathematical calculation algorithms implemented in is this type of codes, the provided solutions are quite precise for a relatively small grid size, with practically negligible computing times. The proposed model is applied to illustrative problems, providing hydraulic isopotential and stream lines, showing that as the dam penetration depth increases, the hydraulic gradient downstream decreases, thus reducing the risk of hydraulic failure.

\section{KEYWORDS}

geotechnics, network model, seepage

\section{1 | INTRODUCTION}

The steady flow of groundwater in an aquifer with partially penetrating retaining structures, such as dams, is governed by a Laplace-type equation. Analytical or semi-analytical solutions ${ }^{1,2}$ can be obtained for a small number of seepage problems, mainly due to the geometric complexity of the domain..$^{3-5}$ Thus, Harr ${ }^{6}$ solved some simple hydraulic structures, and $\mathrm{Mandel}^{7}$ developed a mapping technique to solve analytically seepage related to excavations and cofferdams. In this context, making use of commercial codes, numerical solutions become a viable and precise alternative to obtain the maps (flow net construction) of the stream function (or flow lines, along which the water particles travel from upstream to downstream) and the hydraulic potential (isopotential lines), the main variables of interest. ${ }^{8,9}$ These maps, which provide a clear view of the flow and hydraulic head distribution throughout the domain, represent two orthogonal families of curves, since equipotential lines intersect flow lines at right angles.

In this communication, based on the network simulation method, ${ }^{10}$ a network model for an aquifer area is designed. By combining elements according to the geometry and properties of the domain, and adding the electrical devices that implement the boundary conditions, the complete model can be run in a free circuit resolution code such as Ngspice. ${ }^{11}$ Isotropic (as regards the hydraulic conductivity) soils are assumed. Since the soil is saturated, changes at boundary conditions adjust quickly, so only steady-state solution is of interest. In most cases, the geometry of the typical scenario is a rectangular 2-D domain, whose upper surface is composed by two parts: upstream and downstream. The dam structure 
is assumed impervious. The method goes beyond the scope of the classical electric analogy that is currently used in many textbooks of different engineering fields, particularly in heat transfer, ${ }^{12,13}$ since it is able to work with nonlinear and coupled problems, with applications on problems related with elasticity ${ }^{14}$ and soil consolidation, ${ }^{15}$ among others. In this way, the network method is presented as a very interesting alternative to other numerical methods, given that, in practice, it is only necessary to know some simple programming rules and, in particular, there is a wide variety of free codes that allow to solve complex engineering problems without having to spend large amounts of money on software licenses.

\section{2 | MATHEMATICAL MODEL}

The starting point for the design of the network model is the finite-difference differential equation that comes from the spatial discretization of the governing equation, which can be expressed in terms of the hydraulic head as the dependent variable. As in the method of lines, time is considered a continuous variable, and the flow conservation is assumed directly from the Kirchhoff's law relative to the conservation of the electric current in the circuit network. Both the hydraulic head and the stream function, which are two important variables in engineering, can be derived from each other by simple mathematical routines, but alternatively, it is possible to design models for both quantities and join their solutions in the same section.

A basic scheme of the physical problem is presented in Figure 1. On a porous aquifer, a concrete structure separates two finite regions with different hydraulic head, causing a groundwater flow from the higher water level (left) to the lower one (right), that is, from upstream to downstream. Under the hypotheses of incompressible fluid, no volume changes in the soil and assuming an isotropic hydraulic conductivity (coefficient of permeability $k$ ), the governing equation in terms of total head $(h)$ is obtained by substituting Darcy's law $\left(v_{x}=k \frac{\partial h}{\partial x}\right.$ and $\left.v_{y}=k \frac{\partial h}{\partial y}\right)$ in the continuity equation $\left(\frac{\partial v_{x}}{\partial x}+\frac{\partial v_{y}}{\partial y}=0\right)$. This leads to

$$
k_{x}\left(\frac{\partial^{2} h}{\partial x^{2}}\right)+k_{y}\left(\frac{\partial^{2} h}{\partial y^{2}}\right)=0
$$

In terms of the hydraulic potential, whose relation with $h$ is given by $\phi=\mathrm{kh}+$ constant, it is immediate to establish the governing equation in terms of this scalar function, $\frac{\partial^{2} \phi}{\partial x^{2}}+\frac{\partial^{2} \phi}{\partial y^{2}}=0$, which is also a Laplace-type equation. Since 2-D Laplace equation is satisfied by two conjugate harmonic functions $\phi$ and $\Psi$, so that the curves $\phi(x, y)=$ constant are the orthogonal trajectories of the curves $\Psi(x, y)=$ constant, the last represents the path that water particles follow. $\Psi(x, y)$ is called the stream function, a scalar-type function from which the flow velocity components can be derived, according to the expressions $v_{x}=\frac{\partial \Psi}{\partial y}$ and $v_{y}=-\frac{\partial \Psi}{\partial x}$.

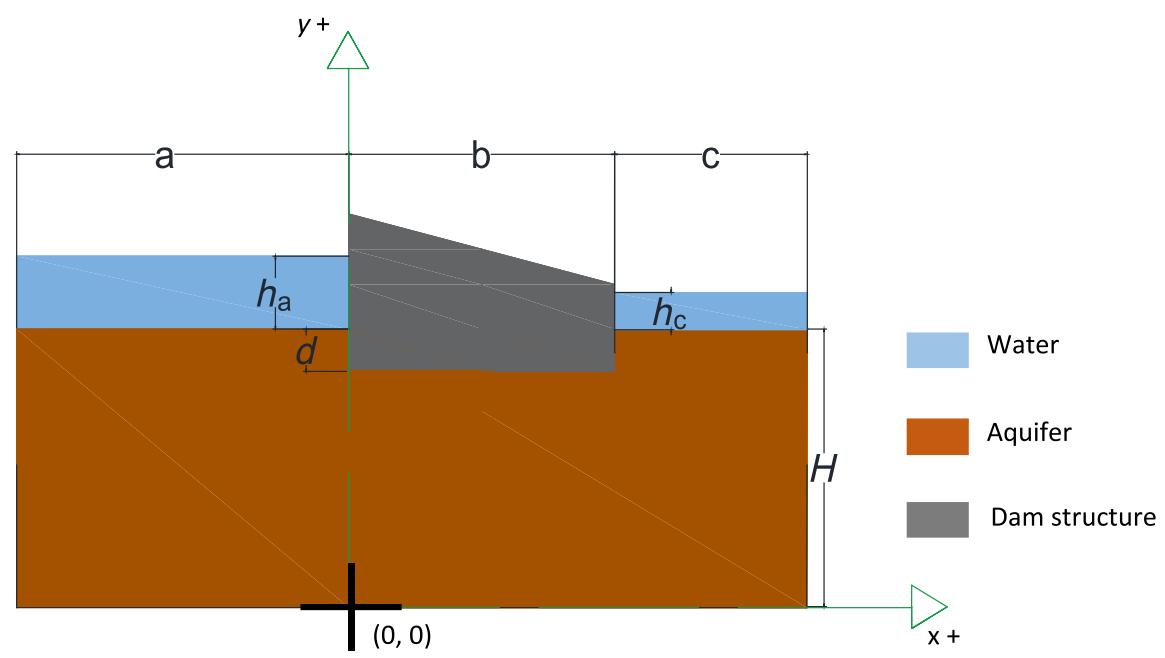

FIGURE 1 Physical representation of the problem 
In terms of the nonindependent functions $\phi$ and $\Psi$, the mathematical model is defined by the following equations ${ }^{16}$ :

$$
\begin{aligned}
& \left(\frac{\partial^{2} \phi}{\partial x^{2}}\right)+\left(\frac{\partial^{2} \phi}{\partial y^{2}}\right)=0 \quad \text { governing equation, } \\
& v_{x}=\frac{\partial \phi}{\partial x}=0 \text { and } \phi=h_{a} \text { at }(x \leq 0, y=H), \\
& v_{x}=\frac{\partial \phi}{\partial x}=0 \text { and } \phi=h_{c} \text { at }(x \geq b, y=H), \\
& v_{x}=\frac{\partial \phi}{\partial x}=0 \text { at }(x=-a, y) \text { and }(x=b+c, y), \\
& v_{y}=\frac{\partial \phi}{\partial y}=0 \text { at }(x, y=0), \\
& v_{x}=\frac{\partial \phi}{\partial x}=v_{y}=\frac{\partial \phi}{\partial y}=0 \text { at }(x=0, H-d \leq y \leq H),(x=b, H-d \leq y \leq H),
\end{aligned}
$$

and

$$
\begin{aligned}
& k_{x}\left(\frac{\partial^{2} \Psi}{\partial x^{2}}\right)+k_{y}\left(\frac{\partial^{2} \Psi}{\partial y^{2}}\right)=0 \text { governing equation, } \\
& \frac{\partial \Psi}{\partial y}=0 \text { at }(x \leq 0, y=H) \text { and }(x \geq b, y=H), \\
& \frac{\partial \Psi}{\partial y}=0 \text { and } \Psi=0 \text { at }(x=-a, y) \text { and }(x=b+c, y), \\
& \frac{\partial \Psi}{\partial x}=0 \text { and } \Psi=0 \text { at }(x, y=0), \\
& \frac{\partial \Psi}{\partial y}=\frac{\partial \Psi}{\partial x}=0 \text { and } \Psi=Q \text { at }(x=0, H-d \leq y \leq H),(x=b, H-d \leq y \leq H), \\
& \quad \text { and }(0 \leq x \leq b, y=H-d),
\end{aligned}
$$

where $Q$ (total infiltrated flow) is obtained after solving the equations for the hydraulic potential ((2a) to (2f)). Boundary conditions ((2b) and (2c)) represent Dirichlet or first-type conditions, since $\phi$ is constant (its value upstream and downstream are different, as it depends on the hydraulic head) along the prescribed boundary; while (2d), (2e), and (2f) are homogeneous Neumann or second-type conditions since the changes of $\phi$ are zero along these boundaries (impervious aquifer limits and impervious structure, respectively). The same criteria have been applied to the rest of equations ((3b) to (3e)). Keep in mind that, since $\phi$ and $\Psi$ are conjugate functions, their respective boundary conditions change to be from one type to the other.

At this point, it is important to keep in mind that, in the methodology proposed in this article, the problem is first solved for the equipotential lines (Equations (2a) to (2f)), from which these are obtained (as well as the infiltrated flow) and, afterwards, is solved the flow lines map (Equations (3a)-(3e)), demonstrating that, actually, the functions $\phi$ and $\Psi$ are decoupled. Thus, the shape of the flow lines only depends on the geometry and the aquifer properties, and only the numerical value of these is proportional to the infiltrated flow.

\section{3 | NETWORK MODEL}

The network simulation method is based on an electrohydraulic analogy that establishes a formal equivalence between the potential variables of the physical problem and the electrical potential of the network model. In addition, water flow velocity and electric current are analogous magnitudes. This equivalence can be established due to the fact that the finite-difference differential equations of the physical and electrical models are the same. Since there are two mathematical models (one for each variable, $\phi$ and $\Psi$ ), two independent circuits are designed, and as the governing equations (2a) 


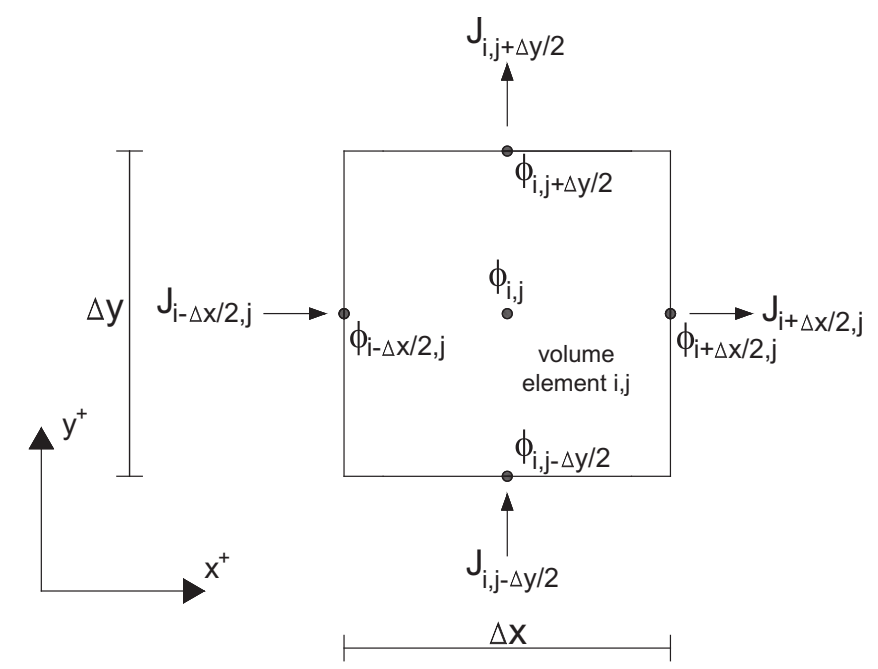

FIGURE 2 Nomenclature for the elementary cell

and (3a) are quite similar, the network models of the elementary cells have the same electrical components. The complete models only differ in the electrical components that are required to implement the boundary conditions. Following the nomenclature of Figure 2, for the element nodes (central node and edge nodes), the spatial discretized equation that derives from (2a) writes as

$$
\frac{\left(\frac{\phi_{i+\frac{\Delta x}{2}, j}-\phi_{i, j}}{\frac{\Delta x}{2}}\right)-\left(\frac{\phi_{i, j}-\phi_{i-\frac{\Delta x}{2}, j}}{\frac{\Delta x}{2}}\right)}{\Delta \mathrm{x}}+\frac{\left(\frac{\phi_{i, j+\frac{\Delta y}{2}}-\phi_{i, j}}{\frac{\Delta y}{2}}\right)-\left(\frac{\phi_{i, j}-\phi_{i, j-\frac{\Delta y}{2}}}{\frac{\Delta y}{2}}\right)}{\Delta \mathrm{y}}=0 .
$$

This equation can be interpreted as the balance between four currents $\left(J_{i, j+\frac{\Delta y}{2}}, J_{i, j-\frac{\Delta y}{2}}, J_{i+\frac{\Delta x}{2}, j}\right.$, and $\left.J_{i-\frac{\Delta x}{2}, j}\right)$ that converge at the center of the element. These currents are defined in the following form:

$$
J_{i+\frac{\Delta x}{2}, j}=\frac{\phi_{i+\frac{\Delta x}{2}, j}-\phi_{i, j}}{\frac{(\Delta x)^{2}}{2}}, \quad J_{i-\frac{\Delta x}{2}, j}=\frac{\phi_{i, j}-\phi_{i-\frac{\Delta x}{2}, j}}{\frac{(\Delta x)^{2}}{2}}, \quad J_{i, j+\frac{\Delta y}{2}}=\frac{\phi_{i, j+\frac{\Delta y}{2}}-\phi_{i, j}}{\frac{(\Delta y)^{2}}{2}}, \quad J_{i, j-\frac{\Delta y}{2}}=\frac{\phi_{i, j}-\phi_{i, j-\frac{\Delta y}{2}}}{\frac{(\Delta y)^{2}}{2}} .
$$

In its electrical analogy, the above equations simulate the current through resistors, of the following values:

$$
R_{i+\frac{\Delta x}{2}, j}=R_{i-\frac{\Delta x}{2}, j}=\frac{(\Delta x)^{2}}{2} \text { and } R_{i, j+\frac{\Delta y}{2}}=R_{i, j-\frac{\Delta y}{2}}=\frac{(\Delta y)^{2}}{2} .
$$

Therefore, the network model for the elementary cell is the one shown in Figure 3. Once the spatial domain is discretized in $N_{\mathrm{x}} \times N_{\mathrm{y}}$ elements and its nodes are joined by ideal electrical contacts, the complete model needs to implement the boundary conditions. To do this, constant value sources are used for the first-type conditions, while infinite value resistors are used for the second type. Network model for the $\Psi$ variable is designed following the same steps as for the $\phi$ variable.

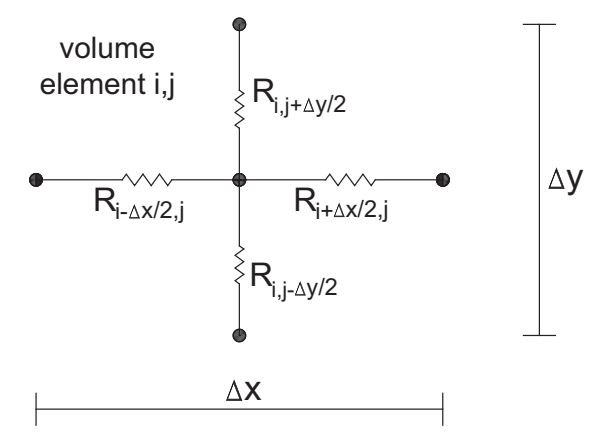

FIGURE 3 Network model of the elementary cell 


\section{4 | APPLiCATIONS OF NETWORK MODEL SOLUTION}

According to the nomenclature of Figure 1, the scenario to be solved has the following parameters: $H=20 \mathrm{~m}$ (aquifer thickness), $b=20 \mathrm{~m}$ (dam width), $a=20 \mathrm{~m}$ (upstream stretch length), $c=20 \mathrm{~m}$ and $40 \mathrm{~m}$ (downstream stretch length), and $d=5 \mathrm{~m}$ and $10 \mathrm{~m}$ (dam penetration depth), giving rise to four different scenarios (I to IV). In what concerns to hydraulic head values, it was considered $10 \mathrm{~m}\left(h_{\mathrm{a}}\right)$ for the upstream level and $0 \mathrm{~m}\left(h_{\mathrm{c}}\right)$ for the downstream level. Finally, it has been assumed that the hydraulic conductivity is $10^{-7} \mathrm{~m} / \mathrm{s}$. Once the above parameters have been introduced into the governing equations, the numerical network model is designed, following the procedure described in the previous section. With the help of a free circuit resolution code such as Ngspice, the complete model can be run, obtaining the solution of the fields of voltages (for functions $\phi$ and $\Psi$ ), in all nodes (points) of the network (aquifer). Finally, these output voltages are conveniently processed by means of simple matrix operations in order to obtain the hydraulic head and flow lines maps. The total computation time is of the order of 5 minutes in an Intel Core i7-6700 CPU 4.00-GHz computer, for a mesh of approximately $120 \times 40$ cells.

Figures 4 to 7 show the seepage maps (functions $\phi$ and $\Psi$ ) for the four scenarios described above. As can be seen, the equipotential lines of the function $\phi($ or $h)$ and the flow lines $(\Psi)$ intersect perpendicularly, which shows that the proposed network model solves the seepage problem precisely, since, as it was said before, the functions $\phi$ and $\Psi$ represent two

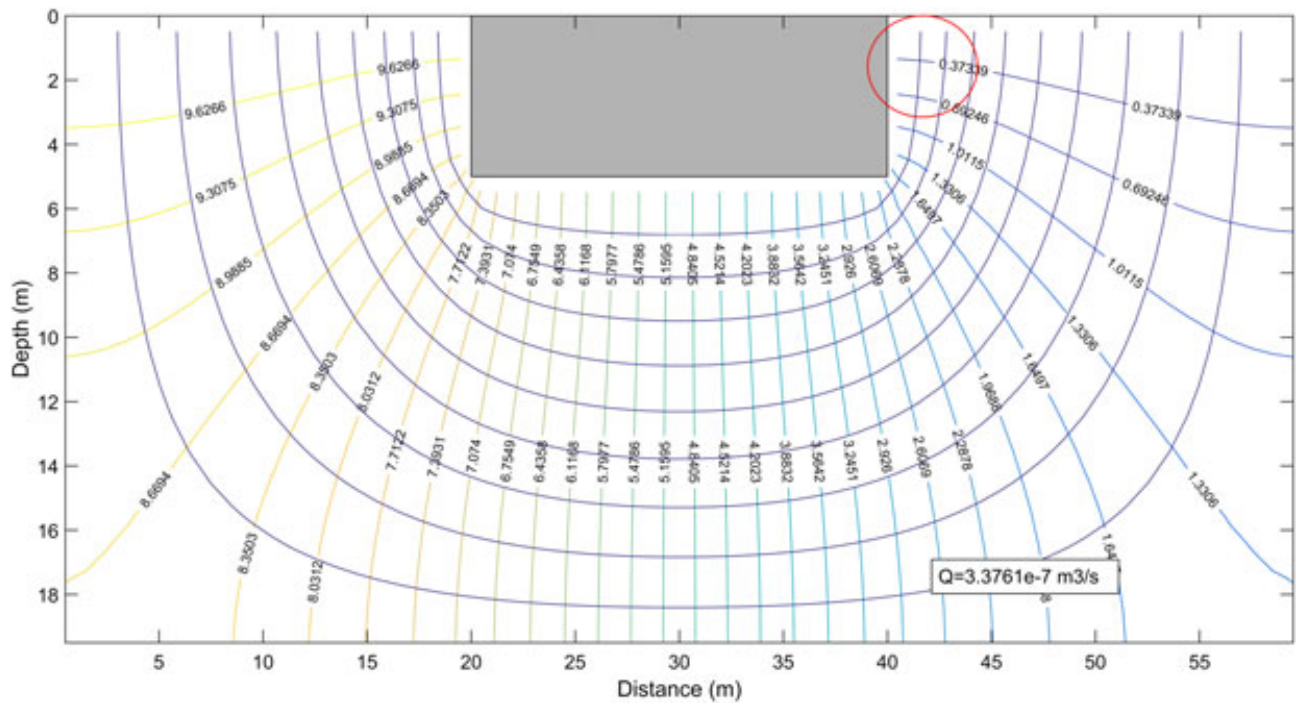

FIGURE 4 Scenario I seepage map. $H=20 \mathrm{~m}, b=20 \mathrm{~m}, a=20 \mathrm{~m}, c=20 \mathrm{~m}$, and $d=5 \mathrm{~m}$

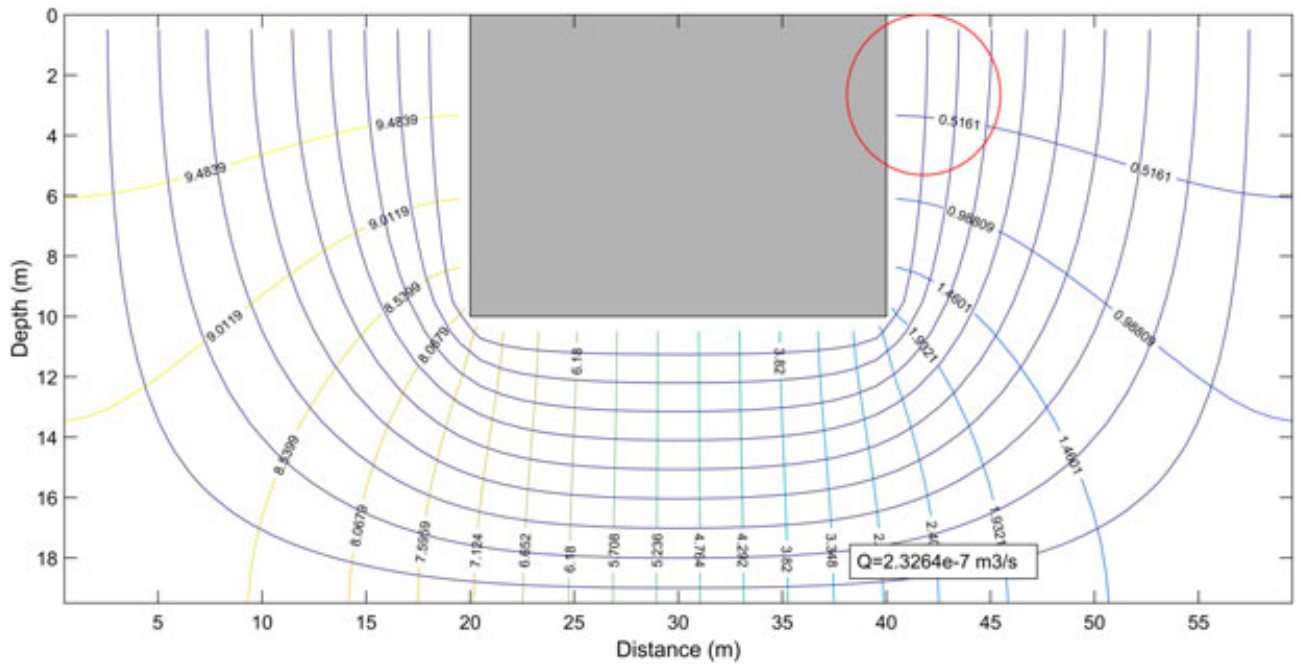

FIGURE 5 Scenario II seepage map. $H=20 \mathrm{~m}, b=20 \mathrm{~m}, a=20 \mathrm{~m}, c=20 \mathrm{~m}$, and $d=10 \mathrm{~m}$ 


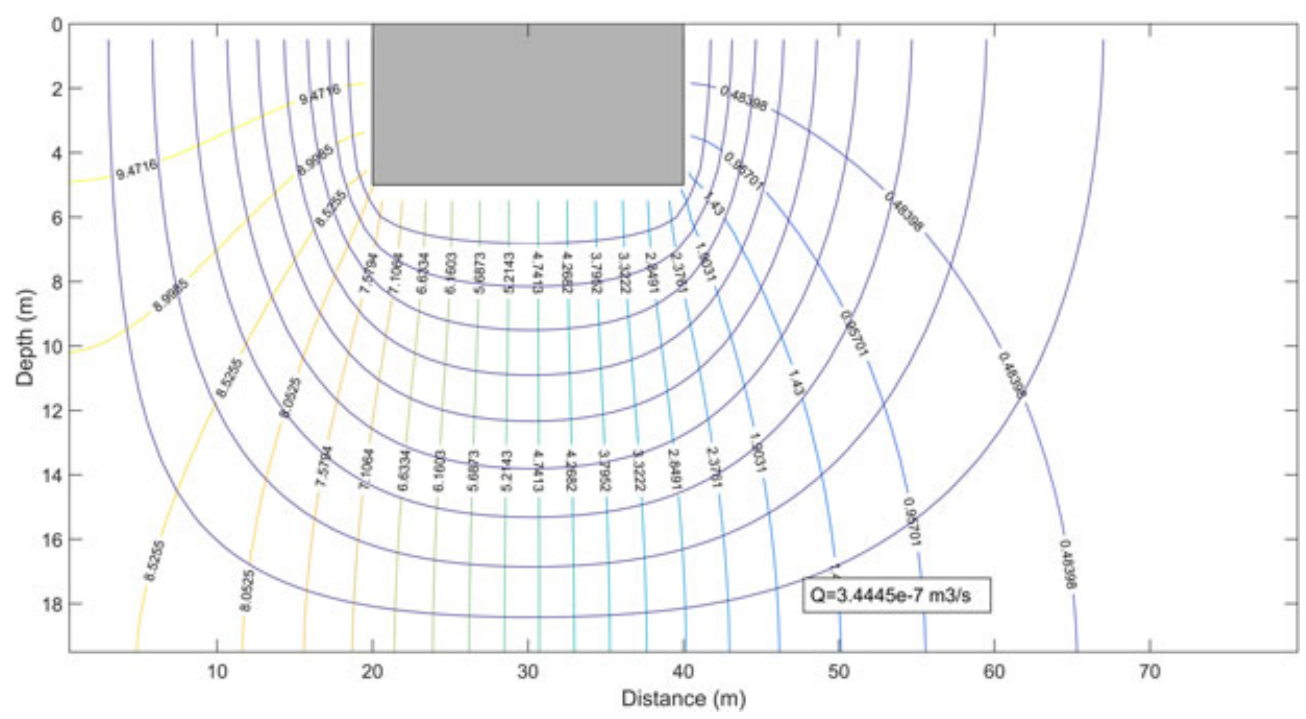

FIGURE 6 Scenario III seepage map. $H=20 \mathrm{~m}, b=20 \mathrm{~m}, a=20 \mathrm{~m}, c=40 \mathrm{~m}$, and $d=5 \mathrm{~m}$

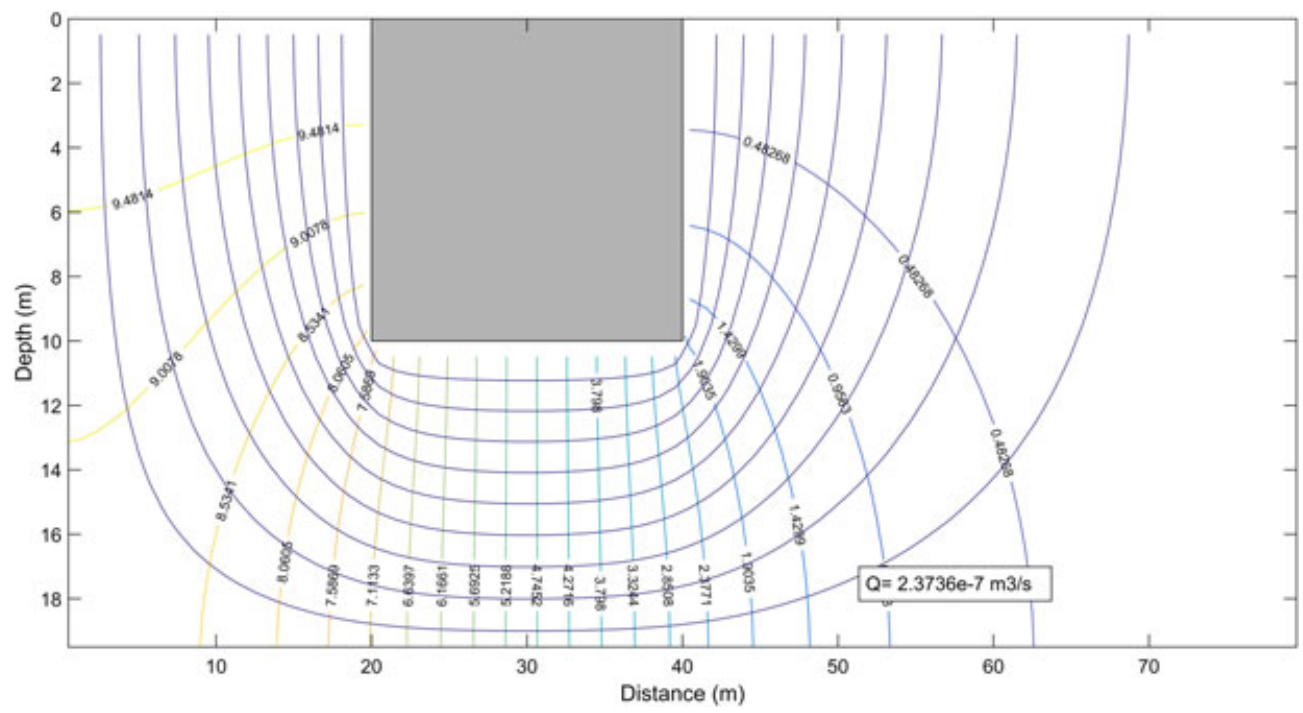

FIGURE 7 Scenario IV seepage map. $H=20 \mathrm{~m}, b=20 \mathrm{~m}, a=20 \mathrm{~m}, c=40 \mathrm{~m}$, and $d=10 \mathrm{~m}$

orthogonal families of curves. To solve these scenarios, a grid of $120 \times 40$ cells has turned out to be enough, showing the power, reliability, and versatility of the method. Even when reducing the mesh grid to $60 \times 20$ cells, the relative errors committed are below $1 \%$, compared with analytical solutions.

In view of the results, it is observed that an increase in the penetration depth of the dam produces a decrease in the hydraulic gradient downstream of the dam, (Figures 4 and 5) which helps to reduce the risk of hydraulic failure, especially adjacent to the dam structure (where the gradients are higher). On the other hand, it can be observed that, in general, an increase of the downstream stretch length leads to a reduction of the hydraulic gradient throughout this area (comparisons between Figures 4 and 6 and Figures 5 and 7), which means that the extension of the model affects the results.

\section{5 | CONCLUSIONS}

The 2-D problem of the groundwater flow under a partially penetrating impervious structure in porous and isotropic aquifers has been simulated and solved by the network method numerical tool. After defining the governing equations of the problem, both in terms of the hydraulic head and the flow lines function (and their respective boundary conditions), 
the network model was designed following the rules of the network method and starting from the spatial discretization of the partial differential equations that govern these variables. Afterwards, by means of a free resolution code of electrical circuits, the numerical calculation of the problem was carried out, providing the solutions for both the equipotential and the flow lines. Finally, through a series of scenarios, it was verified that the solutions obtained with the network method are highly accurate and reliable, being practically identical to those obtained by means of complex analytical expressions.

\section{ACKNOWLEDGEMENTS}

We would like to thank the SéNeCa Foundation for the support given to this research and for the scholarships awarded to María Encarnación Martínez Moreno to carry out her doctoral thesis.

\section{ORCID}

\section{Gonzalo García-Ros (D) https://orcid.org/0000-0002-2837-7358}

\section{REFERENCES}

1. Tracy FT. Using analytic solution methods on unsaturated seepage flow computations. Procedia Comput Sci. 2016;80:554-564.

2. Ilyinsky NB, Kacimov AR, Yakimov ND. Analytical solutions of seepage theory problems. Inverse method, variational theorems, optimization and estimates (a review). Fluid Dyn. 1998;33(2):157-168.

3. Azizi F. Applied Analyses in Geotechnics. Boca Raton, FL: CRC Press; 1999.

4. Lande CK, Bansal RK, Warke A. Simulation of 2-dimensional subsurface seepage flow in an anisotropic porous medium. Perspect Sci. 2016;8:276-278.

5. Bereslavskii EN. The design of the iso-velocity contour for the flow past the base of a dam with a confining bed. J Appl Math Mech. 2009;73(4):426-433.

6. Harr ME. Groundwater and Seepage. New York, NY: McGraw-Hill; 1962.

7. Mandel J. Ecoulement de l'eau sous Une ligne de palplanches: abaque pour la condition de renard. Travaux. 1951;197:273-282.

8. Berry PL, Reid D. An Introduction to Soil Mechanics. New York, NY: McGraw-Hill; 1988.

9. Das BM. Advanced Soil Mechanics. New York, NY: Taylor \& Francis; 2008.

10. González-Fernández CF. Applications of the network simulation method to transport processes. In: Horno J, ed. Network Simulation Method. Trivandrum, India: Research Signpost; 2002.

11. Ngspice open source simulator [software]. http://ngspice.sourceforge.net/. Published 2014.

12. Alhama F, Marín F, Moreno JA. An efficient and reliable model to simulate microscopic mechanical friction in the Frenkel-Kontorova-Tomlinson model. Comput Phys Commun. 2011;182:2314-2325.

13. Alhama I, Soto A, Alhama F. Simulador de flujo y transporte FATSIM-A. Cartagena, Spain: Polytechnic University of Cartagena; 2010.

14. Morales JL, Moreno JA, Alhama F. Application of the network method to simulate elastostatic problems defined by potential functions. Applications to axisymmetrical hollow bodies. Int J Comput Math. 2012;89(13-14):1781-1793.

15. García-Ros G, Alhama I, Morales JL. Numerical simulation of nonlinear consolidation problems by models based on the network method. App Math Model. 2019;69:604-620.

16. Martínez-Moreno E, Alhama I, García-Ros G. Numerical simulation of seepage maps under dams with sheet piles on their ends. Comp and Math Methods. 2019;1:e1010

\section{AUTHOR BIOGRAPHIES}

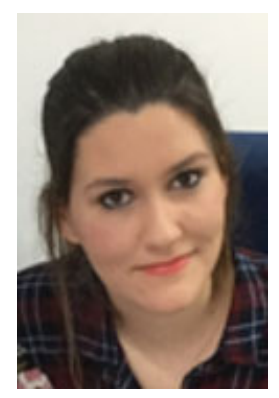

Encarnación Martínez-Moreno is a PhD student with a scholarship at the Higher Technical School of Civil Engineering, Technical University of Cartagena, Cartagena, Spain. She has a bachelor's degree and a master degree in civil engineering. Her research lines are seepage, EPS Geofoam, and geotechnics. 


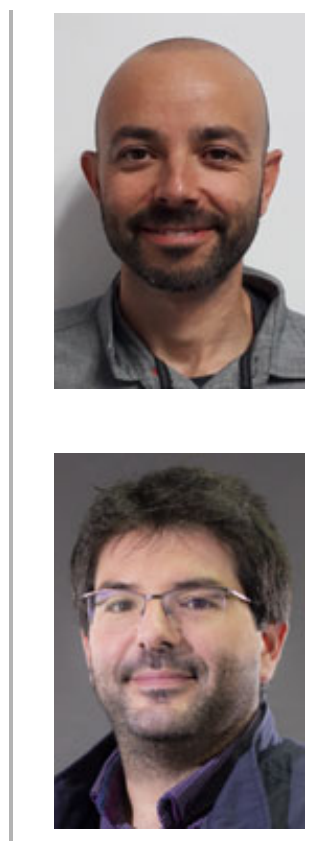

Iván Alhama Manteca is a full professor (ground engineering area) at the Higher Technical School of Civil Engineering, Technical University of Cartagena, Cartagena, Spain. He has a bachelor's degree and a PhD degree in geological sciences. His research lines are seepage, soil consolidation, EPS Geofoam, and geotechnics.

Gonzalo García-Ros is an associate professor (ground engineering area) at the Higher Technical School of Civil Engineering, Technical University of Cartagena, Cartagena, Spain. He has a master degree and a $\mathrm{PhD}$ degree in civil engineering. His research lines are soil consolidation, seepage, creeping, and geotechnics.

How to cite this article: Martínez-Moreno E, Alhama Manteca I, García-Ros G. Network model for the numerical solution of groundwater flow: Application to partially penetrating retaining structures in geotechnical engineering. Comp and Math Methods. 2019;1:e1039. https://doi.org/10.1002/cmm4.1039 